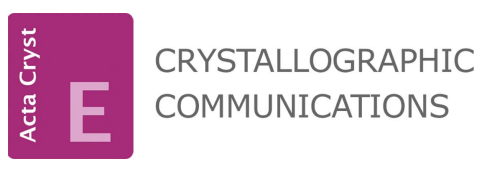

ISSN 2056-9890

Received 3 September 2019

Accepted 30 September 2019

Edited by A. J. Lough, University of Toronto, Canada

Keywords: crystal structure; polymorphism; oxonium cation; sulfonate anions.

CCDC references: 1956689; 1956690

Supporting information: this article has supporting information at journals.iucr.org/e

\section{The crystal structures of two novel polymorphs of bis(oxonium) ethane-1,2-disulfonate}

\author{
Jaroslaw Mazurek* and Ana Fernandez-Casares
}

Ardena, Solid State Research Services, Meibergdreef 31, 1105 AZ Amsterdam, The Netherlands. *Correspondence e-mail:
jaroslaw.mazurek@ardena.com

Two novel crystal forms of bis(oxonium) ethane-1,2-disulfonate, $2 \mathrm{H}_{3} \mathrm{O}^{-}$.$\mathrm{C}_{2} \mathrm{H}_{4} \mathrm{O}_{6} \mathrm{~S}_{2}{ }^{2-}$, are reported. Polymorph II has monoclinic $\left(P 2_{1} / n\right)$ symmetry, while the symmetry of form III is triclinic $(P \overline{1})$. Both structures display extensive networks of $\mathrm{O}-\mathrm{H} \cdots \mathrm{O}$ hydrogen bonds. While this network in Form II is similar to that observed for the previously reported Form I [Mootz \& Wunderlich (1970). Acta Cryst. B26, 1820-1825; Sartori et al. (1994). Z. Naturforsch. 49, 1467-1472] and extends in all directions, in Form III it differs significantly, forming layers parallel to the $a b$ plane. The sulfonate molecule in all three forms adopts a nearly identical geometry. The other observed differences between the forms, apart from the hydrogen-bonding network, are observed in the crystal density and packing index.

\section{Chemical context}

Sulfonic acids are commonly used in salt formation in the pharmaceutical industry, especially for poorly or non soluble in water drugs (Neau \& Loka, 2018). Salts of ethane-1,2-disulfonic acid account for $0.38 \%$ of all the FDA-approved commercially marketed salts (Steele \& Talbir, 2016) and therefore its toxicology, dosage (Saal \& Becker, 2013) and various physico-chemical properties are widely studied (Black et al., 2007; Elder et al., 2010). In our laboratory, ethane-1,2disulfonic acid is commonly used in the salt screening for increasing solubility as well as improving the crystallinity of various researched active pharmaceutical ingredients (APIs).<smiles>O=S(=O)([O-])CCS(=O)(=O)[O-]</smiles>

\section{Structural commentary}

The sulfonate anion in all polymorphs, including the previously determined form (Mootz \& Wunderlich, 1970, refcode HOEDSO; Sartori et al., 1994, refcode HOEDSO01) has a nearly identical geometry. In all cases, the center of the $\mathrm{C}-\mathrm{C}$ bond is located on an inversion center, and the $\mathrm{C}-\mathrm{S}$ and $\mathrm{C}-\mathrm{O}$ distances in all cases are within $3 \sigma$. The sulfonate group adopts the geometry of an open umbrella with the $\mathrm{C}-\mathrm{S}-\mathrm{O}$ bond angles of $106.51(6), 105.82(6), 107.23(6)^{\circ}$ for Form II (Fig. 1) and 106.16 (11), 106.21 (10), $107.20(12)^{\circ}$ for Form III 


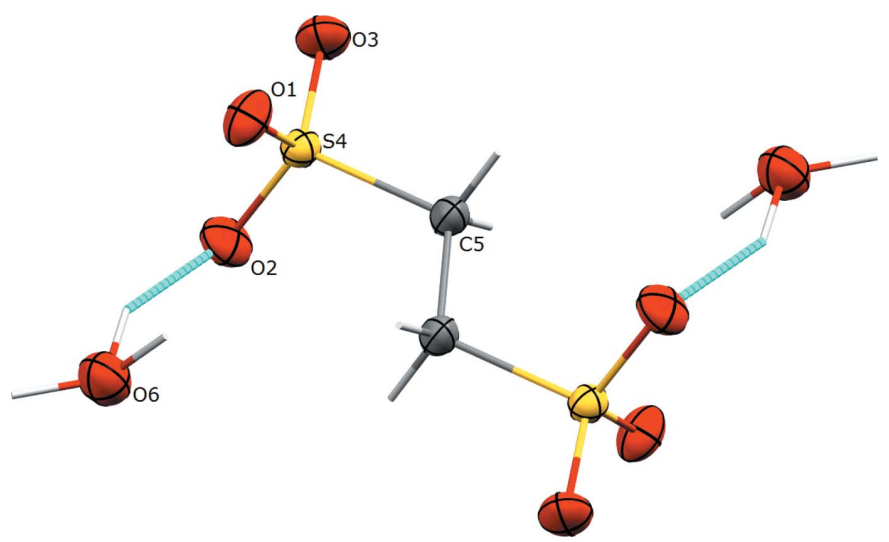

Figure 1

The molecular structure of an anion-cation pair of Form II, with the atom-labelling scheme. Displacement ellipsoids are drawn at the $50 \%$ probability level and hydrogen bonds are shown in torquoise. Unlabelled atoms are related to labelled ones by the symmetry operator $(-x+1$, $-y+1,-z+1)$.

(Fig. 2). The values of all $\mathrm{O}-\mathrm{S}-\mathrm{O}$ angles are above $110^{\circ}$ [112.91 (7), 111.48 (7), 112.37 (7) ${ }^{\circ}$ for Form II and 111.31 (11), $113.45(11), 112.00(12)^{\circ}$ for Form III]. In this way, the molecular symmetry of the sulfonate group becomes slightly distorted $C_{3 V}$. In all crystals, the oxonium cations have a pyramidal geometry with slightly elongated $\mathrm{O}-\mathrm{H}$ distances for one $\mathrm{H}$ atom. This is most likely an effect of the fast exchange of a proton ( $\mathrm{H}$ atom) between the sulfonate group and the water molecules.

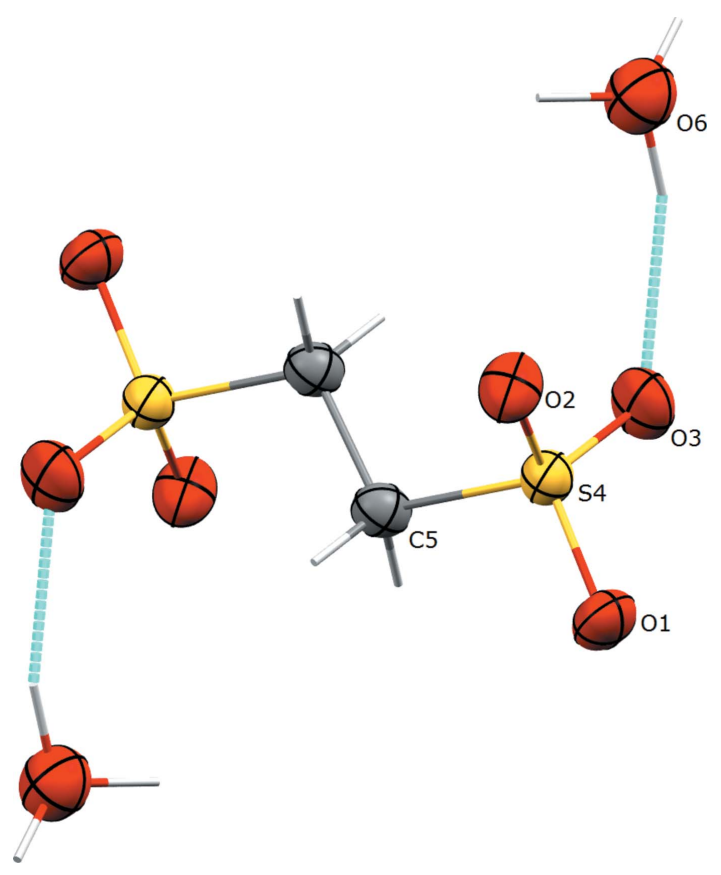

Figure 2

The molecular structure of anion cation pair of Form III, with the atomlabelling scheme. Displacement ellipsoids are drawn at the $50 \%$ probability level and hydrogen bonds are shown in torquoise. Unlabelled atoms are related to labelled ones by the symmetry operator $(-x+1$, $-y+1,-z+1)$.
Table 1

Hydrogen-bond geometry $\left(\AA,^{\circ}\right)$ for Form II.

\begin{tabular}{|c|c|c|c|c|}
\hline$D-\mathrm{H} \cdots A$ & $D-\mathrm{H}$ & $\mathrm{H} \cdots A$ & $D \cdots A$ & $D-\mathrm{H} \cdots A$ \\
\hline $\mathrm{O} 6-\mathrm{H} 63 \cdots \mathrm{O} 2$ & $0.99(2)$ & $2.62(2)$ & 3.1795 (17) & $116(2)$ \\
\hline $\mathrm{O} 6-\mathrm{H} 61 \cdots \mathrm{O} 2^{\mathrm{i}}$ & $1.00(2)$ & $2.02(3)$ & $2.9312(16)$ & $150(3)$ \\
\hline $\mathrm{O} 6-\mathrm{H} 62 \cdots \mathrm{O} 3$ & $1.06(2)$ & $1.92(3)$ & $2.9141(16)$ & $154(3)$ \\
\hline $\mathrm{O} 6-\mathrm{H} 61 \cdots \mathrm{O}^{\mathrm{ii}}$ & $1.00(2)$ & $2.60(3)$ & $2.9857(16)$ & $103(2)$ \\
\hline $\mathrm{O} 6-\mathrm{H} 63 \cdots \mathrm{O} 1^{\mathrm{iii}}$ & $0.99(2)$ & $2.14(2)$ & $3.0266(18)$ & $148(2)$ \\
\hline
\end{tabular}

Symmetry codes: (i) $-x+\frac{3}{2}, y+\frac{1}{2},-z+\frac{1}{2}$; (ii) $-x+1,-y+2,-z+1$; (iii) $x+1, y, z$.

Table 2

Hydrogen-bond geometry $\left(\AA,^{\circ}\right)$ for Form III.

\begin{tabular}{lllll}
\hline$D-\mathrm{H} \cdots A$ & $D-\mathrm{H}$ & $\mathrm{H} \cdots A$ & $D \cdots A$ & $D-\mathrm{H} \cdots A$ \\
\hline O6-H61 $\cdots \mathrm{O} 1^{\mathrm{i}}$ & $1.07(4)$ & $1.93(4)$ & $2.991(3)$ & $170(4)$ \\
$\mathrm{O}^{\mathrm{ii}}-\mathrm{H} 62 \cdots \mathrm{O} 2^{\mathrm{i}}$ & $1.02(4)$ & $2.52(3)$ & $3.002(3)$ & $108(2)$ \\
$\mathrm{O}^{-}-\mathrm{H} 62 \cdots \mathrm{O} 3$ & $1.02(4)$ & $1.97(4)$ & $2.945(3)$ & $158(3)$ \\
$\mathrm{O}^{\mathrm{O}}-\mathrm{H} 63 \cdots \mathrm{O} 1^{\mathrm{iii}}$ & $1.02(4)$ & $1.89(4)$ & $2.899(3)$ & $173(3)$ \\
\hline
\end{tabular}

Symmetry codes: (i) $x, y-1, z$; (ii) $x-1, y, z$; (iii) $x-1, y-1, z$.

The biggest differences between forms are observed in the density of the crystal, as well as in the packing coefficient (Kitajgorodskij, 1973). The lowest values of both parameters are attributed to Form III ( $1.60 \mathrm{~g} \mathrm{~cm}^{-3}$ and 0.67 , respectively), which suggests that this polymorph is the least stable. Form II presented here has a slightly better packing index than previously reported for Form I (Mootz \& Wunderlich, 1970; Sartori et al., 1994) 0.75 versus 0.73 . On the other hand, the density is lower: 1.78 versus $1.82 \mathrm{~g} \mathrm{~cm}^{-3}$, respectively.

\section{Supramolecular features}

The hydrogen bonds between the oxonium cations and sulfonate anions in the crystal of Form II (Table 1, Fig. 3) extend in all directions forming a three-dimensional network similar to that observed for Form I (Mootz \& Wunderlich, 1970; Sartori et al., 1994). However, contrary to the previously

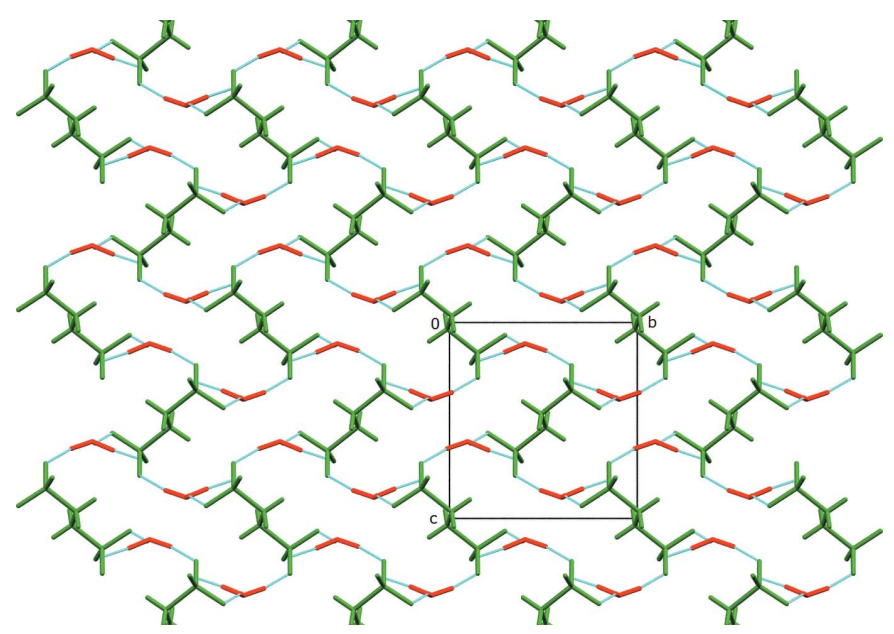

Figure 3

The crystal packing of Form II, viewed along the $a$ axis. The ethane-1,2disulfonate dianions are coloured in green, while oxonium cations are red and hydrogen bonds are shown in torquoise. 
Table 3

Experimental details.

Form II

Crystal data

Chemical formula

$M_{\mathrm{r}}$

Crystal system, space group

Temperature (K)

$a, b, c(\AA)$

$\alpha, \beta, \gamma\left({ }^{\circ}\right)$

$V\left(\AA^{3}\right)$

$Z$

Radiation type

$\mu\left(\mathrm{mm}^{-1}\right)$

Crystal size (mm)

Data collection

Diffractometer

Absorption correction

$T_{\min }, T_{\max }$

No. of measured, independent and observed $[I>$ $2 \sigma(I)]$ reflections

$R_{\text {int }}$

$(\sin \theta / \lambda)_{\max }\left(\AA^{-1}\right)$

Refinement

$R\left[F^{2}>2 \sigma\left(F^{2}\right)\right], w R\left(F^{2}\right), S$

No. of reflections

No. of parameters

No. of restraints

$\mathrm{H}$-atom treatment

$\Delta \rho_{\max }, \Delta \rho_{\min }\left(\mathrm{e} \AA^{-3}\right)$

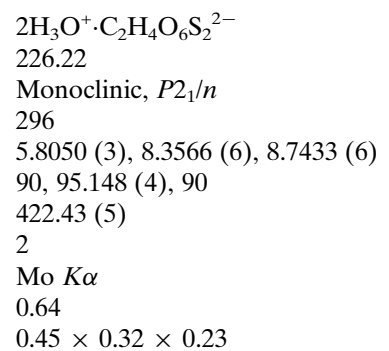

Bruker KappaCCD

Gaussian integration (Coppens, 1970)

$0.748,0.907$

$17906,1848,1768$

0.075

0.806

$0.041,0.121,1.04$

1848

76

3

All $\mathrm{H}$-atom parameters refined

$0.62,-0.93$
Form III

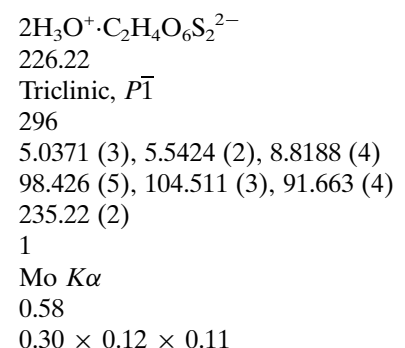

Bruker KappaCCD

Gaussian integration (Coppens, 1970) $0.813,0.947$

$7504,1708,1192$

0.131

0.758

$0.058,0.163,1.04$

1708

76

0

All $\mathrm{H}$-atom parameters refined $0.66,-0.67$

Computer programs: COLLECT (Hooft, 1998), HKL SCALEPACK and DENZO (Otwinowski \& Minor, 1997), SHELXT (Sheldrick, 2015a), SHELXL2014/7 (Sheldrick, 2015b), Mercury (Macrae et al., 2008) and enCIFer (Allen et al., 2004).

reported form, where the hydrogen-bond network is built from alternate anion-cations layers, in Form II such layers could not be distinguished. The supramolecular behaviour of Form III is significantly different. In this case (Table 2 and Fig. 4), the anion-cation hydrogen-bond network forms separate layers parallel to the $a b$ plane built from sulfonate anions surrounded by oxonium cations with no interactions between the planes.

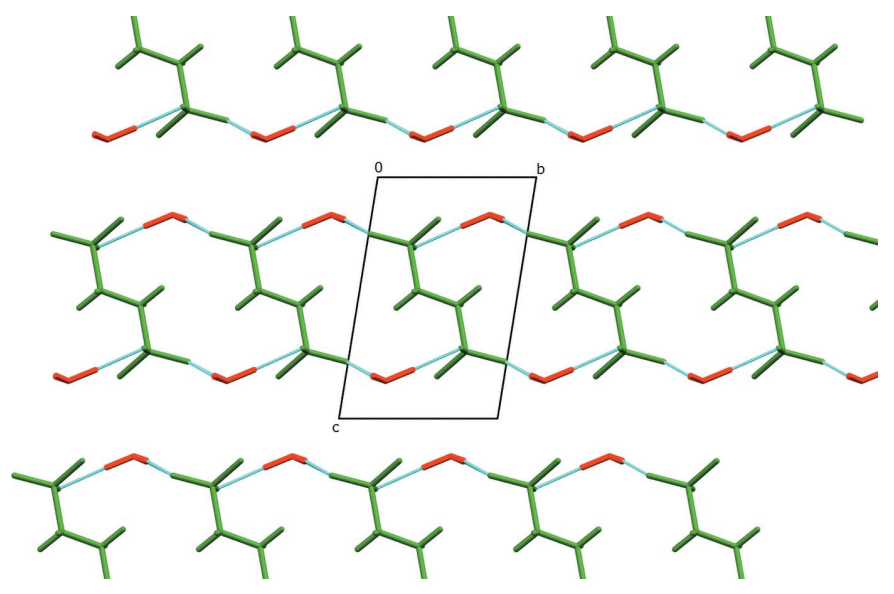

Figure 4

The crystal packing of Form III, viewed along the $a$ axis. The ethane-1,2disulfonate dianions are coloured in green, while oxonium cations are red and hydrogen bonds are shown in turquoise.

\section{Database survey}

As mentioned above, the crystal structure of a different polymorphic form of oxonium ethane-1,2-disulfonate has been previously reported (Mootz, \& Wunderlich, 1970, refcode HOEDSO; Sartori et al., 1994, refcode HOEDSO01). Apart from these structures, there are 12 hits for ethane-1,2-disulfonate salts in the Cambridge Structural Database (CSD, Version 5.40; ConQuest 2.02; Groom et al., 2016), one of which is disordered. The geometry of the sulfonate group in all of the anions is nearly the same, with slightly distorted $C_{3 v}$ molecular symmetry for the open-umbrella geometry. The average values of the $\mathrm{C}-\mathrm{S}-\mathrm{O}$ and $\mathrm{O}-\mathrm{S}-\mathrm{O}$ bond angles are very close to those reported in this paper: $105.9 \pm 0.8$ and $112.8 \pm 0.9^{\circ}$, respectively.

\section{Synthesis and crystallization}

Both crystals were obtained from an aqueous solution during unsuccessful salt formation with an unnamed free base (API) in water. Firstly, columnar crystals of Form III that appeared to be unstable were grown from the thick oil and within time transformed into prismatic crystals of Form II.

\section{Refinement}

Crystal data, data collection and structure refinement details are summarized in Table 3. All $\mathrm{H}$ atoms were found in 
difference-Fourier maps and refined with isotropic displacement parameters. The DFIX 0.980 .03 O6 H61, O6 H62 and O6 H63 instruction in SHELXL2014/7 (Sheldrick, 2015b) was used to restrain the oxonium $\mathrm{O}-\mathrm{H}$ distance in Form II. All of the oxonium $\mathrm{H}$ atoms in Form III were refined independently without any restraints.

\section{References}

Allen, F. H., Johnson, O., Shields, G. P., Smith, B. R. \& Towler, M. (2004). J. Appl. Cryst. 37, 335-338.

Black, S. N., Collier, E. A., Davey, R. J. \& Roberts, R. J. (2007). J. Pharm. Sci. 96, 1053-1068.

Coppens, P. (1970). Crystallographic Computing, edited by F. R. Ahmed, S. R. Hall \& C. P. Huber, pp. 255-270. Copenhagen: Munksgaard.

Elder, D. P., Delaney, E. D., Teasdale, A., Eyley, S., Reif, V. D., Jacq, K., Facchine, K. L., Oestrich, R. S., Sandra, P. \& David, F. (2010). J. Pharm. Sci. 99, 2948-2961.

Groom, C. R., Bruno, I. J., Lightfoot, M. P. \& Ward, S. C. (2016). Acta Cryst. B72, 171-179.
Hooft, R. W. W. (1998). COLLECT. Nonius BV, Delft, The Netherlands.

Kitajgorodskij, A. I. (1973). Molecular Crystals and Molecules. New York: Academic Press.

Macrae, C. F., Bruno, I. J., Chisholm, J. A., Edgington, P. R., McCabe, P., Pidcock, E., Rodriguez-Monge, L., Taylor, R., van de Streek, J. \& Wood, P. A. (2008). J. Appl. Cryst. 41, 466-470.

Mootz, D. \& Wunderlich, H. (1970). Acta Cryst. B26, 1820-1825.

Neau, S. H. \& Loka, N. C. (2018). 15 Pharmaceutical Salts. In WaterInsoluble Drug Formulation, edited by R. Liu, pp. 451-469. Boca Raton: CRC Press.

Otwinowski, Z. \& Minor, W. (1997). Methods in Enzymology, Vol. 276, Macromolecular Crystallography, Part A, edited by C. W. Carter Jr \& R. M. Sweet, pp. 307-326. New York: Academic Press. Saal, C. \& Becker, A. (2013). Eur. J. Pharm. Sci. 49, 614-623.

Sartori, P., Jüschke, R., Boese, R. \& Bläser, D. (1994). Z. Naturforsch. 49, 1467-1472.

Sheldrick, G. M. (2015a). Acta Cryst. A71, 3-8.

Sheldrick, G. M. (2015b). Acta Cryst. C71, 3-8.

Steele, G. \& Talbir, A. (2016). In Pharmaceutical preformulation and formulation, edited by M. Gibson, pp. 29-140. Boca Raton: CRC Press. 


\section{supporting information}

Acta Cryst. (2019). E75, 1586-1589 [https://doi.org/10.1107/S2056989019013367]

\section{The crystal structures of two novel polymorphs of bis(oxonium) ethane-1,2-di- sulfonate}

\section{Jaroslaw Mazurek and Ana Fernandez-Casares}

\section{Computing details}

For both structures, data collection: COLLECT (Hooft, 1998); cell refinement: HKL SCALEPACK (Otwinowski \& Minor, 1997); data reduction: HKL DENZO and SCALEPACK (Otwinowski \& Minor, 1997); program(s) used to solve structure: SHELXT (Sheldrick, 2015a); program(s) used to refine structure: SHELXL2014/7 (Sheldrick, 2015b); molecular graphics: Mercury (Macrae et al., 2008); software used to prepare material for publication: enCIFer (Allen et al., 2004).

Bis(oxonium) ethane-1,2-disulfonate (I)

\section{Crystal data}

$2 \mathrm{H}_{3} \mathrm{O}^{+} \cdot \mathrm{C}_{2} \mathrm{H}_{4} \mathrm{O}_{6} \mathrm{~S}_{2}^{2-}$

$M_{r}=226.22$

Monoclinic, $P 2_{1} / n$

$a=5.8050(3) \AA$

$b=8.3566(6) \AA$

$c=8.7433(6) \AA$

$\beta=95.148(4)^{\circ}$

$V=422.43(5) \AA^{3}$

$Z=2$

\section{Data collection}

Bruker KappaCCD diffractometer

Radiation source: fine-focus sealed tube

Horizonally mounted graphite crystal monochromator

Detector resolution: 9 pixels $\mathrm{mm}^{-1}$

CCD scans

Absorption correction: integration

Gaussian integration (Coppens, 1970)

Refinement

Refinement on $F^{2}$

Least-squares matrix: full

$R\left[F^{2}>2 \sigma\left(F^{2}\right)\right]=0.041$

$w R\left(F^{2}\right)=0.121$

$S=1.04$

1848 reflections

76 parameters

3 restraints
$F(000)=236$

$D_{\mathrm{x}}=1.778 \mathrm{Mg} \mathrm{m}^{-3}$

Mo $K \alpha$ radiation, $\lambda=0.71073 \AA$

Cell parameters from 11538 reflections

$\theta=1.0-35.0^{\circ}$

$\mu=0.64 \mathrm{~mm}^{-1}$

$T=296 \mathrm{~K}$

Prism, pale yellow

$0.45 \times 0.32 \times 0.23 \mathrm{~mm}$

$T_{\min }=0.748, T_{\max }=0.907$

17906 measured reflections

1848 independent reflections

1768 reflections with $I>2 \sigma(I)$

$R_{\text {int }}=0.075$

$\theta_{\max }=34.9^{\circ}, \theta_{\min }=3.4^{\circ}$

$h=-9 \rightarrow 9$

$k=-13 \rightarrow 13$

$l=-14 \rightarrow 14$

Primary atom site location: difference Fourier map

Secondary atom site location: difference Fourier map

Hydrogen site location: difference Fourier map

All H-atom parameters refined

$w=1 /\left[\sigma^{2}\left(F_{\mathrm{o}}^{2}\right)+(0.0797 P)^{2}+0.1864 P\right]$

where $P=\left(F_{\mathrm{o}}{ }^{2}+2 F_{\mathrm{c}}{ }^{2}\right) / 3$

$(\Delta / \sigma)_{\max }=0.026$ 
$\Delta \rho_{\max }=0.62$ e $\AA^{-3}$

$\Delta \rho_{\min }=-0.93$ e $\AA^{-3}$
Extinction correction: SHELXL-2014/7

(Sheldrick 2015b),

$\mathrm{Fc}^{*}=\mathrm{kFc}\left[1+0.001 \times \mathrm{Fc}^{2} \lambda^{3} / \sin (2 \theta)\right]^{-1 / 4}$

Extinction coefficient: $0.20(2)$

Special details

Geometry. All esds (except the esd in the dihedral angle between two 1.s. planes) are estimated using the full covariance matrix. The cell esds are taken into account individually in the estimation of esds in distances, angles and torsion angles; correlations between esds in cell parameters are only used when they are defined by crystal symmetry. An approximate (isotropic) treatment of cell esds is used for estimating esds involving l.s. planes.

Fractional atomic coordinates and isotropic or equivalent isotropic displacement parameters $\left(\AA^{2}\right)$

\begin{tabular}{lllll}
\hline & $x$ & $y$ & $z$ & $U_{\text {iso }} * / U_{\text {eq }}$ \\
\hline O1 & $0.03777(17)$ & $0.61584(15)$ & $0.29236(14)$ & $0.0319(2)$ \\
O2 & $0.4298(2)$ & $0.65073(13)$ & $0.21941(13)$ & $0.0302(2)$ \\
O3 & $0.30307(19)$ & $0.79554(12)$ & $0.43552(13)$ & $0.0308(2)$ \\
S4 & $0.27802(4)$ & $0.64978(3)$ & $0.34467(3)$ & $0.01890(13)$ \\
C5 & $0.3753(2)$ & $0.48838(15)$ & $0.46678(14)$ & $0.0231(2)$ \\
H5A & $0.270(5)$ & $0.476(3)$ & $0.541(3)$ & $0.043(6)^{*}$ \\
H5B & $0.360(4)$ & $0.393(3)$ & $0.404(2)$ & $0.025(5)^{*}$ \\
O6 & $0.7708(2)$ & $0.90258(15)$ & $0.39286(16)$ & $0.0363(3)$ \\
H61 & $0.822(6)$ & $1.009(3)$ & $0.357(4)$ & $0.066(9)^{*}$ \\
H62 & $0.588(4)$ & $0.889(4)$ & $0.383(4)$ & $0.067(9)^{*}$ \\
H63 & $0.797(4)$ & $0.795(3)$ & $0.350(3)$ & $0.041(7)^{*}$ \\
\hline
\end{tabular}

Atomic displacement parameters $\left(\AA^{2}\right)$

\begin{tabular}{lllllll}
\hline & $U^{11}$ & $U^{22}$ & $U^{33}$ & $U^{12}$ & $U^{13}$ & $U^{23}$ \\
\hline O1 & $0.0219(4)$ & $0.0345(5)$ & $0.0373(5)$ & $-0.0002(3)$ & $-0.0079(4)$ & $0.0012(4)$ \\
O2 & $0.0341(5)$ & $0.0339(5)$ & $0.0238(4)$ & $0.0083(4)$ & $0.0096(4)$ & $0.0055(3)$ \\
O3 & $0.0333(5)$ & $0.0227(4)$ & $0.0362(5)$ & $0.0020(3)$ & $0.0025(4)$ & $-0.0081(4)$ \\
S4 & $0.01863(17)$ & $0.01917(17)$ & $0.01865(17)$ & $0.00184(7)$ & $0.00025(10)$ & $0.00054(7)$ \\
C5 & $0.0218(4)$ & $0.0231(5)$ & $0.0234(5)$ & $-0.0027(3)$ & $-0.0032(3)$ & $0.0061(4)$ \\
O6 & $0.0307(5)$ & $0.0320(5)$ & $0.0452(6)$ & $-0.0022(4)$ & $-0.0012(4)$ & $0.0000(5)$
\end{tabular}

Geometric parameters $\left(\AA,{ }^{o}\right)$

\begin{tabular}{llll}
\hline $\mathrm{O} 1-\mathrm{S} 4$ & $1.4561(10)$ & $\mathrm{C} 5-\mathrm{H} 5 \mathrm{~A}$ & $0.94(3)$ \\
$\mathrm{O} 2-\mathrm{S} 4$ & $1.4658(11)$ & $\mathrm{C} 5-\mathrm{H} 5 \mathrm{~B}$ & $0.97(2)$ \\
$\mathrm{O} 3-\mathrm{S} 4$ & $1.4544(10)$ & $\mathrm{O} 6-\mathrm{H} 61$ & $1.00(2)$ \\
$\mathrm{S} 4-\mathrm{C} 5$ & $1.7804(11)$ & $\mathrm{O} 6-\mathrm{H} 62$ & $1.06(2)$ \\
$\mathrm{C} 5-\mathrm{C} 5$ & $\mathrm{O} 6-\mathrm{H} 63$ & $0.99(2)$ \\
& $1.523(2)$ & & $108.0(16)$ \\
$\mathrm{O} 3-\mathrm{S} 4-\mathrm{O} 1$ & & $\mathrm{~S} 4-\mathrm{C} 5-\mathrm{H} 5 \mathrm{~A}$ & $110.7(12)$ \\
$\mathrm{O} 3-\mathrm{S} 4-\mathrm{O} 2$ & $112.37(7)$ & $\mathrm{C} 5-\mathrm{C} 5-\mathrm{H} 5 \mathrm{~B}$ & $106.3(12)$ \\
$\mathrm{O} 1-\mathrm{S} 4-\mathrm{O} 2$ & $111.48(7)$ & $\mathrm{S} 4-\mathrm{C} 5-\mathrm{H} 5 \mathrm{~B}$ & $105(2)$ \\
$\mathrm{O} 3-\mathrm{S} 4-\mathrm{C} 5$ & $112.91(7)$ & $\mathrm{H} 5 \mathrm{~A}-\mathrm{C} 5-\mathrm{H} 5 \mathrm{~B}$ & $113(3)$ \\
$\mathrm{O} 1-\mathrm{S} 4-\mathrm{C} 5$ & $107.23(6)$ & $\mathrm{H} 61-\mathrm{O} 6-\mathrm{H} 62$ &
\end{tabular}




$\begin{array}{llll}\mathrm{O} 2-\mathrm{S} 4-\mathrm{C} 5 & 105.82(6) & \mathrm{H} 61-\mathrm{O} 6-\mathrm{H} 63 & 129(2) \\ \mathrm{C} 5-\mathrm{C} 5-\mathrm{S} 4 & 111.82(11) & \mathrm{H} 62-\mathrm{O} 6-\mathrm{H} 63 & 93(2) \\ \mathrm{C} 5-\mathrm{C} 5-\mathrm{H} 5 \mathrm{~A} & 114.1(17) & & \\ & & & \\ \mathrm{O} 3-\mathrm{S} 4-\mathrm{C} 5-\mathrm{C} 5^{\mathrm{i}} & -57.98(14) & \mathrm{O} 2-\mathrm{S} 4-\mathrm{C} 5-\mathrm{C} 5^{\mathrm{i}} & \\ \mathrm{O} 1-\mathrm{S} 4-\mathrm{C} 5-\mathrm{C} 5^{\mathrm{i}} & -178.48(12) & & \end{array}$

Symmetry code: (i) $-x+1,-y+1,-z+1$.

Hydrogen-bond geometry $\left(\AA,{ }^{\circ}\right)$

\begin{tabular}{lllll}
\hline$D-\mathrm{H} \cdots A$ & $D-\mathrm{H}$ & $\mathrm{H} \cdots A$ & $D \cdots A$ & $D-\mathrm{H} \cdots A$ \\
\hline $\mathrm{O} 6-\mathrm{H} 63 \cdots \mathrm{O} 2$ & $0.99(2)$ & $2.62(2)$ & $3.1795(17)$ & $116(2)$ \\
$\mathrm{O} 6-\mathrm{H} 61 \cdots \mathrm{O} 2^{\mathrm{ii}}$ & $1.00(2)$ & $2.02(3)$ & $2.9312(16)$ & $150(3)$ \\
$\mathrm{O} 6-\mathrm{H} 62 \cdots \mathrm{O} 3$ & $1.06(2)$ & $1.92(3)$ & $2.9141(16)$ & $154(3)$ \\
$\mathrm{O}^{-}-\mathrm{H} 61 \cdots \mathrm{O} 3{ }^{\mathrm{iii}}$ & $1.00(2)$ & $2.60(3)$ & $2.9857(16)$ & $103(2)$ \\
$\mathrm{O}^{\mathrm{ii}}-\mathrm{H} 63 \cdots \mathrm{O} 1$ & $0.99(2)$ & $2.14(2)$ & $3.0266(18)$ & $148(2)$
\end{tabular}

Symmetry codes: (ii) $-x+3 / 2, y+1 / 2,-z+1 / 2$; (iii) $-x+1,-y+2,-z+1$; (iv) $x+1, y, z$.

Bis(oxonium) ethane-1,2-disulfonate (II)

Crystal data

$2 \mathrm{H}_{3} \mathrm{O}^{+} \cdot \mathrm{C}_{2} \mathrm{H}_{4} \mathrm{O}_{6} \mathrm{~S}_{2}{ }^{2-}$

$M_{r}=226.22$

Triclinic, $P \overline{1}$

$a=5.0371(3) \AA$

$b=5.5424(2) \AA$

$c=8.8188(4) \AA$

$\alpha=98.426(5)^{\circ}$

$\beta=104.511(3)^{\circ}$

$\gamma=91.663(4)^{\circ}$

$V=235.22(2) \AA^{3}$

\section{Data collection}

Bruker KappaCCD

diffractometer

Radiation source: fine-focus sealed tube

Horizonally mounted graphite crystal monochromator

Detector resolution: 9 pixels $\mathrm{mm}^{-1}$

CCD scans

Absorption correction: integration

Gaussian integration (Coppens, 1970)

Refinement

Refinement on $F^{2}$

Least-squares matrix: full

$R\left[F^{2}>2 \sigma\left(F^{2}\right)\right]=0.058$

$w R\left(F^{2}\right)=0.163$

$S=1.04$

1708 reflections

76 parameters

0 restraints
$Z=1$

$F(000)=118$

$D_{\mathrm{x}}=1.597 \mathrm{Mg} \mathrm{m}^{-3}$

Mo $K \alpha$ radiation, $\lambda=0.71073 \AA$

Cell parameters from 4728 reflections

$\theta=1.0-32.6^{\circ}$

$\mu=0.57 \mathrm{~mm}^{-1}$

$T=296 \mathrm{~K}$

Columnar, colorless

$0.30 \times 0.12 \times 0.11 \mathrm{~mm}$

$T_{\min }=0.813, T_{\max }=0.947$

7504 measured reflections

1708 independent reflections

1192 reflections with $I>2 \sigma(I)$

$R_{\text {int }}=0.131$

$\theta_{\max }=32.6^{\circ}, \theta_{\min }=2.4^{\circ}$

$h=-7 \rightarrow 6$

$k=-8 \rightarrow 8$

$l=-11 \rightarrow 13$

Primary atom site location: difference Fourier map

Secondary atom site location: difference Fourier map

Hydrogen site location: difference Fourier map

All H-atom parameters refined

$w=1 /\left[\sigma^{2}\left(F_{\mathrm{o}}^{2}\right)+(0.0869 P)^{2}+0.0186 P\right]$

where $P=\left(F_{\mathrm{o}}^{2}+2 F_{\mathrm{c}}^{2}\right) / 3$ 
$(\Delta / \sigma)_{\max }=0.016$

$\Delta \rho_{\max }=0.66 \mathrm{e} \AA^{-3}$

$\Delta \rho_{\min }=-0.67$ e $\AA^{-3}$
Extinction correction: SHELXL-2014/7

(Sheldrick 2015b),

$\mathrm{Fc}^{*}=\mathrm{kFc}\left[1+0.001 \mathrm{xFc}^{2} \lambda^{3} / \sin (2 \theta)\right]^{-1 / 4}$

Extinction coefficient: $0.19(3)$

\section{Special details}

Geometry. All esds (except the esd in the dihedral angle between two 1.s. planes) are estimated using the full covariance matrix. The cell esds are taken into account individually in the estimation of esds in distances, angles and torsion angles; correlations between esds in cell parameters are only used when they are defined by crystal symmetry. An approximate (isotropic) treatment of cell esds is used for estimating esds involving l.s. planes.

Fractional atomic coordinates and isotropic or equivalent isotropic displacement parameters $\left(\AA^{2}\right)$

\begin{tabular}{lllll}
\hline & $x$ & $y$ & $z$ & $U_{\text {iso }} * / U_{\text {eq }}$ \\
\hline O1 & $0.6030(4)$ & $0.9888(3)$ & $0.7642(2)$ & $0.0387(4)$ \\
O2 & $0.6622(4)$ & $0.5760(3)$ & $0.8252(2)$ & $0.0378(4)$ \\
O3 & $0.2119(4)$ & $0.6995(4)$ & $0.7086(3)$ & $0.0417(5)$ \\
S4 & $0.50463(11)$ & $0.73087(9)$ & $0.72171(6)$ & $0.0267(2)$ \\
C5 & $0.5533(5)$ & $0.6324(4)$ & $0.5302(3)$ & $0.0290(5)$ \\
H5A & $0.473(7)$ & $0.750(6)$ & $0.459(4)$ & $0.043(8)^{*}$ \\
H5B & $0.737(7)$ & $0.654(6)$ & $0.526(4)$ & $0.054(9)^{*}$ \\
O6 & $0.1360(5)$ & $0.2554(4)$ & $0.8443(3)$ & $0.0477(5)$ \\
H61 & $0.318(10)$ & $0.173(8)$ & $0.827(5)$ & $0.083(13)^{*}$ \\
H62 & $0.113(7)$ & $0.415(6)$ & $0.798(5)$ & $0.049(9)^{*}$ \\
H63 & $-0.056(8)$ & $0.173(6)$ & $0.822(5)$ & $0.057(10)^{*}$ \\
\hline
\end{tabular}

Atomic displacement parameters $\left(\AA^{2}\right)$

\begin{tabular}{lllllll}
\hline & $U^{11}$ & $U^{22}$ & $U^{33}$ & $U^{12}$ & $U^{13}$ & $U^{23}$ \\
\hline O1 & $0.0405(11)$ & $0.0264(8)$ & $0.0464(10)$ & $-0.0019(7)$ & $0.0115(8)$ & $-0.0030(7)$ \\
O2 & $0.0425(11)$ & $0.0396(9)$ & $0.0301(8)$ & $0.0109(8)$ & $0.0059(7)$ & $0.0062(7)$ \\
O3 & $0.0235(9)$ & $0.0476(10)$ & $0.0538(11)$ & $0.0009(7)$ & $0.0125(8)$ & $0.0028(8)$ \\
S4 & $0.0229(3)$ & $0.0261(3)$ & $0.0297(3)$ & $0.0012(2)$ & $0.0060(2)$ & $0.0014(2)$ \\
C5 & $0.0289(12)$ & $0.0297(11)$ & $0.0271(10)$ & $-0.0025(9)$ & $0.0059(9)$ & $0.0034(8)$ \\
O6 & $0.0415(13)$ & $0.0454(11)$ & $0.0535(12)$ & $0.0046(9)$ & $0.0092(10)$ & $0.0046(9)$
\end{tabular}

Geometric parameters $\left(\AA,{ }^{\circ}\right)$

\begin{tabular}{llll}
\hline $\mathrm{O} 1-\mathrm{S} 4$ & $1.4625(17)$ & $\mathrm{C} 5-\mathrm{H} 5 \mathrm{~A}$ & $0.99(3)$ \\
$\mathrm{O} 2-\mathrm{S} 4$ & $1.4509(18)$ & $\mathrm{C} 5-\mathrm{H} 5 \mathrm{~B}$ & $0.94(4)$ \\
$\mathrm{O} 3-\mathrm{S} 4$ & $1.4532(19)$ & $\mathrm{O} 6-\mathrm{H} 61$ & $1.07(4)$ \\
$\mathrm{S} 4-\mathrm{C} 5$ & $1.777(2)$ & $\mathrm{O} 6-\mathrm{H} 62$ & $1.02(4)$ \\
$\mathrm{C} 5-\mathrm{C} 55^{\mathrm{i}}$ & $1.519(4)$ & $\mathrm{O} 6-\mathrm{H} 63$ & $1.02(4)$ \\
& & & \\
$\mathrm{O} 2-\mathrm{S} 4-\mathrm{O} 3$ & $112.00(12)$ & $\mathrm{S} 4-\mathrm{C} 5-\mathrm{H} 5 \mathrm{~A}$ & $109.0(18)$ \\
$\mathrm{O} 2-\mathrm{S} 4-\mathrm{O} 1$ & $113.45(11)$ & $\mathrm{C} 5-\mathrm{C} 5-\mathrm{H} 5 \mathrm{~B}$ & $113(2)$ \\
$\mathrm{O} 3-\mathrm{S} 4-\mathrm{O} 1$ & $111.31(11)$ & $\mathrm{S} 4-\mathrm{C} 5-\mathrm{H} 5 \mathrm{~B}$ & $98(3)$ \\
$\mathrm{O} 2-\mathrm{S} 4-\mathrm{C} 5$ & $106.21(10)$ & $\mathrm{H} 5 \mathrm{~A}-\mathrm{C} 5-\mathrm{H} 5 \mathrm{~B}$ & $111(3)$ \\
$\mathrm{O} 3-\mathrm{S} 4-\mathrm{C} 5$ & $107.20(12)$ & $\mathrm{H} 61-\mathrm{O} 6-\mathrm{H} 62$ &
\end{tabular}




$\begin{array}{llll}\mathrm{O} 1-\mathrm{S} 4-\mathrm{C} 5 & 106.16(11) & \mathrm{H} 61-\mathrm{O} 6-\mathrm{H} 63 & 127(3) \\ \mathrm{C} 5-\mathrm{C} 5-\mathrm{S} 4 & 111.0(2) & \mathrm{H} 62-\mathrm{O} 6-\mathrm{H} 63 & 107(3) \\ \mathrm{C} 5-\mathrm{C} 5-\mathrm{H} 5 \mathrm{~A} & 114.8(19) & & \\ \mathrm{O} 2-\mathrm{S} 4-\mathrm{C} 5-\mathrm{C} 5^{\mathrm{i}} & 61.3(3) & \mathrm{O} 1-\mathrm{S} 4-\mathrm{C} 5-\mathrm{C} 5^{\mathrm{i}} & -177.6(2) \\ \mathrm{O} 3-\mathrm{S} 4-\mathrm{C} 5-\mathrm{C} 5^{\mathrm{i}} & -58.6(3) & & \end{array}$

Symmetry code: (i) $-x+1,-y+1,-z+1$.

Hydrogen-bond geometry $\left(\AA,{ }^{\circ}\right)$

\begin{tabular}{lllll}
\hline$D-\mathrm{H} \cdots A$ & $D-\mathrm{H}$ & $\mathrm{H} \cdots A$ & $D \cdots A$ & $D-\mathrm{H} \cdots A$ \\
\hline $\mathrm{O} 6-\mathrm{H} 61 \cdots \mathrm{O} 1^{\mathrm{ii}}$ & $1.07(4)$ & $1.93(4)$ & $2.991(3)$ & $170(4)$ \\
$\mathrm{O} 6-\mathrm{H} 62 \cdots \mathrm{O} 2^{\mathrm{iii}}$ & $1.02(4)$ & $2.52(3)$ & $3.002(3)$ & $108(2)$ \\
$\mathrm{O} 6-\mathrm{H} 62 \cdots \mathrm{O} 3$ & $1.02(4)$ & $1.97(4)$ & $2.945(3)$ & $158(3)$ \\
$\mathrm{O} 6-\mathrm{H} 63 \cdots{ }^{\text {iv }}$ & $1.02(4)$ & $1.89(4)$ & $2.899(3)$ & $173(3)$
\end{tabular}

Symmetry codes: (ii) $x, y-1, z$; (iii) $x-1, y, z$; (iv) $x-1, y-1, z$. 